\title{
Perceiving Threat In the Face of Safety: Excitation and Inhibition of Conditioned Fear in Human Visual Cortex
}

\author{
Vladimir Miskovic and Andreas Keil \\ Center for the Study of Emotion and Attention, University of Florida, Gainesville, Florida 32611
}

Previous findings have established that cortical sensory systems exhibit experience-dependent biases toward stimuli consistently associated with threat. It remains unclear whether safety cues also facilitate perceptual engagement or how competition between learned threat and safety cues is resolved within visual cortex. Here, we used classical discrimination conditioning with simple luminance modulated visual stimuli that predicted the presence or absence of an aversive sound to examine visuocortical competition between features signaling threat versus safety. We tracked steady-state visual evoked potentials to label distinct visual cortical responses in humans to conditioned and control stimuli. Trial-by-trial expectancy ratings collected online confirmed that participants discriminated between threat and safety cues. Conditioning was associated with heightened activation of the extended visual cortex in response to the threat, but not the safety, stimulus. Cortical facilitation for the threatening stimulus was selective and not decreased by simultaneously presenting safe and associatively novel cues. Our findings shed light on the sensory brain dynamics associated with experiencedependent acquisition of perceptual biases for danger and safety signals.

\section{Introduction}

Sensory systems are biased toward objects that are familiar and highly relevant to behavior. Converging evidence from electroencephalogram (EEG)/event-related potential (Stolarova et al., 2006; Keil et al., 2007), magnetoencephalogram (MEG) (Moratti and Keil, 2005, 2009; Moratti et al., 2006; Weisz et al., 2007; Kluge et al., 2011), and fMRI (Morris et al., 1998; Armony and Dolan, 2002) indicates that fear conditioning is one, highly efficient, mechanism through which stimuli attain access to preferred processing. Simple (Stolarova et al., 2006; Padmala and Pessoa, 2008) and complex (Damaraju et al., 2009) cues reliably associated with danger facilitate engagement of lower-tier sensory cortices, optimizing perception of threat-related features. An outstanding question is whether learned safety cues are also subject to sensory amplification and how sensory systems respond to a threat cue in the presence of a simultaneous safety signal. Co-occurrence of threat and safety signals is common in natural environments in which danger cues (e.g., growling dog) are perceived in moderating contexts (e.g., presence of a leash).

Recently developed conditional discrimination procedures provide relatively independent estimates of threat and safety conditioning but permit assessment of threat-safety competition via compound stimulus presentations (Myers et al., 2009). Threatsafety compounds have been demonstrated to dampen fear-

\footnotetext{
Received Aug. 1, 2012; revised Sept. 27, 2012; accepted 0ct. 23, 2012.

Author contributions: V.M. and A.K. designed research; V.M. performed research; A.K. contributed unpublished reagents/analytic tools; V.M. and A.K. analyzed data; V.M. and A.K. wrote the paper.

This research was supported by National Institute of Mental Health Grants R01 MH084932-02 and R01 MH097320 awarded to A.K. and a fellowship from the Canadian Institutes of Health Research awarded to V.M.

Correspondence should be addressed to either Vladimir Miskovic or Andreas Keil, Center for the Study of Emotion and Attention, University of Florida, P.0. Box 112766, Gainesville, FL 32611, E-mail: vmiskovic@ufl.edu or akeil@ufl.edu.

DOI:10.1523/JNEUROSCI.3692-12.2013

Copyright $\odot 2013$ the authors $\quad 0270-6474 / 13 / 330072-07 \$ 15.00 / 0$
}

potentiated startle reflexes in humans (Jovanovic et al., 2005) and nonhuman animals (Myers and Davis, 2004; Winslow et al., 2008), suggesting that peripheral defensive mobilization is modulated by a safety cue. The aim of this study was to track shortterm changes in visuocortical biases during threat and safety conditioning and during threat-safety compound viewing.

We recorded steady-state visual evoked potentials (ssVEPs) while participants associated simple visual cues with the presence (threat signal) or absence (safety signal) of an aversive sound. The ssVEP is a large-scale oscillatory field potential whose frequency is determined by entrainment of the visual cortices using periodic luminance modulation (Vialatte et al., 2010). Here we used two different flicker rates for the concurrently displayed threat and safety cues (i.e., frequency tagging; Wang et al., 2007) to independently quantify the strength of visual cortical responses evoked by each respective stimulus. Increased power at a specific ssVEP frequency is taken to indicate enhanced sensory activation in response to the driving stimulus (Andersen and Müller, 2010).

Threat and safety training was followed by a transfer test using threat-safety compounds. The extant literature suggests that visual processing varies with the motivational relevance of a given stimulus (Lang and Bradley, 2010). Based on previous work (Moratti et al., 2006), we therefore expected greater ssVEP enhancement for the conditioned threat cue compared with the safety cue, which in turn was expected to elicit greater ssVEP power than the neutral cues, which had no predictive value and thus less motivational relevance. Alternative hypotheses were examined for the critical transfer test: (1) participants might show attenuated ssVEPs for the threat cue when combined with a safety indicator as suggested by fear-potentiated startle reflex data (Jovanovic et al., 2005); or (2) a visuocortical response bias for threat may persist, insulated from competing elements (Wieser at al., 2011). 
A

\begin{tabular}{|c|c|c|c|}
\hline Habituation & Conditioning & Transfer & Extinction \\
\hline 16 AX/16 BX & $16 \mathrm{AX}+/ 16 \mathrm{BX}-$ & $\mathbf{8 ~ A X + / 8 ~ B X - ~}$ & $\mathbf{8 ~ A X}$ \\
& & $16 \mathrm{AB} / 16 \mathrm{AC}$ & \\
\hline
\end{tabular}

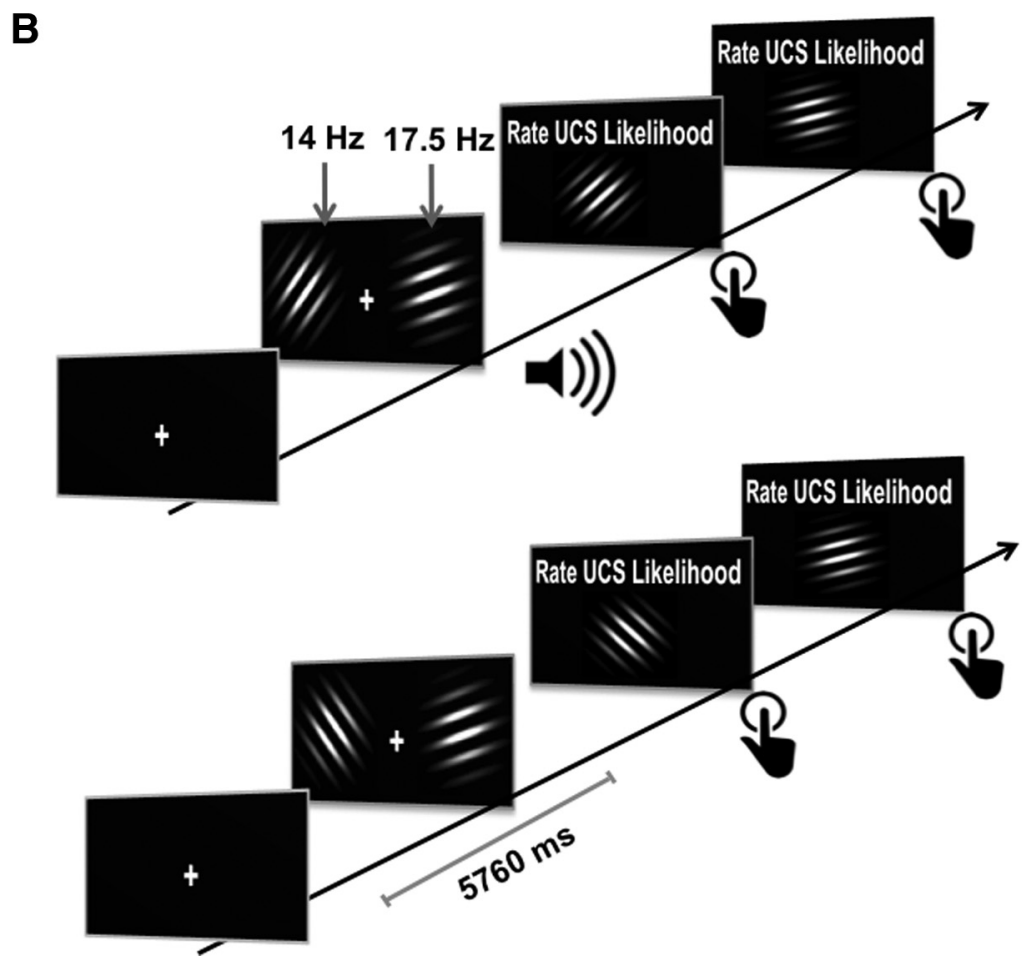

Figure 1. A depicts the basic experimental structure and number of trials included in each of the four phases. Data from the extinction block are not presented in this study because it did not relate to the main questions of interest. A schematic of $A X+$ and $B X-$ trial types in the conditioning phase of the experiment is shown in $B$. Note that the $A+\operatorname{stimulus~}\left(45^{\circ}\right.$ clockwise grating) was paired with the aversive sound on $100 \%$ of the trials, whereas the $B-$ stimulus ( $45^{\circ}$ anticlockwise grating) was never paired with the aversive sound. The visual stimuli were flickered at two distinct frequencies ( 14 and $17.5 \mathrm{~Hz}$ ) with flicker rate assignment for each hemifield being counterbalanced across participants.

\section{Materials and Methods}

Participants. Twenty-nine (mean \pm SD, $18.52 \pm 1.02$ years; 17 females) undergraduate students were initially recruited for study participation in exchange for course credit. Of these 29 subjects, some were excluded for the following reasons: high artifact content in EEG $(n=2)$, request to opt out of the experiment as a result of aversion to the loud sound $(n=2)$, and technical difficulties in sound $(n=1)$ or visual delivery $(n=3)$. After these exclusions, data from a total of 21 participants (11 females) were entered into final analyses.

Stimuli. The visual stimuli (Fig. 1, bottom) were generated using the Psychophysics Toolbox (Brainard, 1997; Pelli, 1997) for MATLAB. They consisted of black-and-white sinusoidal gratings (Gaussian windowed with maximal contrast at center) subtending horizontal and vertical visual angles of $6.5^{\circ}$. The gratings were low in spatial frequency (1.3 cycles $/^{\circ}$ ) and differed from each other only in their degrees of offset from the vertical meridian: $45^{\circ}$ anticlockwise tilt (B cue), $45^{\circ}$ clockwise tilt (A cue), $75^{\circ}$ clockwise tilt (X cue), and $15^{\circ}$ (C cue) clockwise tilt.

The unconditioned stimulus (UCS) was a $1.2 \mathrm{~s}$ white-noise burst, generated in MATLAB and presented through free-field speakers surrounding the participant and set at $92-\mathrm{dB}$ sound pressure level.

Procedure. After providing written informed consent and initial screening to rule out epilepsy, participants were seated in a soundattenuated, dimly lit electrically shielded chamber in which the sensor net was applied. Participants were provided with oral and written instructions. To aid awareness of the appropriate contingency structure, participants were explicitly instructed as follows: "Although you will see pairs of gratings at any time, only one of these gratings will be predicting a loud sound. The angle of one of the gratings — not its brightness, location, size, or any other factor-will predict whether or not you will hear a loud sound. Your task is to learn which angle predicts the loud sound. You will be asked to rate each grating as to how likely it is to be paired with the loud sound, so please pay attention and try to answer as accurately as possible."

Participants were seated at a distance of $1.5 \mathrm{~m}$ from the monitor. The monitor had a $70 \mathrm{~Hz}$ vertical refresh rate with a 19 inch display. The experiment consisted of four separate phases, interleaved with brief $(\sim 1-2 \mathrm{~min}$ ) breaks (Fig. $1 A$ ). All trials were preceded by a $1 \mathrm{~s}$ isolated fixation cross displayed at the center of the screen, which remained on screen for the remainder of the trial. Trials ended with a variable (2-3 s) intertrial interval. Non-reinforced conditioning trials were $5.7 \mathrm{~s}$ in length. The reinforced conditioning trials $(\mathrm{AX}+)$ were $6.9 \mathrm{~s}$ in length, with the extra $1.2 \mathrm{~s}$ added to provide adequate time for the UCS to coterminate with visual stimulus presentation. The additional $1.2 \mathrm{~s}$ of EEG data were excluded from Fourier analysis to avoid contamination of the ssVEP with sound presentation. The gratings presented in left and right hemifields were tagged at different frequencies (14 and $17.5 \mathrm{~Hz}$ ), using on/off cycles, with the tagging order counterbalanced between participants.

The habituation phase involved 16 unreinforced presentations of two cue pairs, AX and BX, with hemifield fully balanced. Participants were explicitly instructed that the habituation phase would not involve UCS presentations. During the conditioning phase, participants were instructed that they may or may not hear a loud white-noise burst and that the angle of one grating stimulus would be the critical predictor. As 
indicated in Figure $1 B$, reinforcement of element $\mathrm{X}$ was conditional on the presence of $\mathrm{A}$ and $\mathrm{B}$ elements during the conditioning phase. Each of the $16 \mathrm{AX}+$ trials coterminated with the UCS (100\% reinforcement schedule), whereas each of the $16 \mathrm{BX}$ - trials predicted UCS absence. Trial order was pseudorandomized such that no more than three reinforced trials were ever presented in succession. The last four trials in the habituation phase and all trials in the conditioning phase were followed ( $2.5 \mathrm{~s}$ later) by separate ratings of each grating presented on that given trial to provide a subjective estimate of grating-UCS pairing likelihood. Participants completed the ratings online by dragging a mouse pointer across the horizontal plane of the screen, to indicate "not likely" (on the left), "uncertain" (middle), or "likely" (right) association of the grating with the UCS.

The beginning of the transfer phase included eight reinforced $\mathrm{AX}+$ trials and eight unreinforced BX - trials, intermixed to provide "booster" learning trials. Immediately after the initial $\mathrm{AX}+/ \mathrm{BX}-$ trials and in the absence of a signaled transition, we included $16 \mathrm{AB}$ and $16 \mathrm{AC}$ unreinforced compound trials. The purpose of the AC trials (in which the $\mathrm{C}$ was an associatively naive cue, not previously encountered) was to control for the possibility that any potential decrement in responding to the excitatory cue on $\mathrm{AB}$ trials might be attributed to the introduction of a novel compound rather than to the safety-related information inherent in the inhibitory cue per se. The final extinction phase was included primarily for ethical reasons, and the findings from this phase will not be formally presented here because they included only half the number of trials contained in the other experimental phases. Before the beginning of the extinction phase, participants were explicitly instructed that there would no longer be any UCS presentations during this phase, which consisted of eight unreinforced AX trials.

The experiment was programmed and controlled using the MATLAB Psychophysics Toolbox (Brainard, 1997; Pelli, 1997). All procedures were approved by the local institutional review board of the University of Florida and were in line with the Declaration of Helsinki.

EEG data recording. EEG was continuously recorded from 257 electrodes using an Electrical Geodesics high-density sensor net, digitized at a rate of $250 \mathrm{~Hz}$, using the vertex sensor $(\mathrm{Cz})$ as the recording reference, with online bandpass filters set at $0.1 \mathrm{~Hz}$ (high pass) and $50 \mathrm{~Hz}$ (low pass). Sensor impedances were kept below $60 \mathrm{k} \Omega$.

EEG data reduction and analyses. Offline EEG analyses were implemented using the EMEGS (ElectroMagnetoEncephalography) toolbox for MATLAB (Peyk et al., 2011). Relative to stimulus onset, epochs were extracted from the raw EEG that included $400 \mathrm{~ms}$ pre-onset and $5700 \mathrm{~ms}$ post-onset for all conditions. Data were filtered using a $20 \mathrm{~Hz}$ low pass (45 dB/octave, 12th-order Butterworth) and a $10 \mathrm{~Hz}$ high pass $(18 \mathrm{~dB} /$ octave, fourth-order Butterworth). As outlined by Junghöfer et al. (2000), statistical parameters were used to find and remove artifactcontaminated channels and trials. The original recording reference $(\mathrm{Cz})$ was first used to detect recording artifacts, and then the data were average referenced to detect global artifacts. Subsequently, bad sensors within individual trials were identified and interpolated based on rejection criteria for amplitude, SD, and gradient. After artifact rejection, an average \pm SD of $82.9 \pm 15.7 \%$ of the trials in the habituation phase, $84.8 \pm$ $14.7 \%$ of the trials in the conditioning phase, and $78 \pm 20.4 \%$ of the trials in the transfer phase were retained. The artifact-free data were then submitted to a discrete Fourier transform with segments extracted from 1400 to $5700 \mathrm{~ms}$ after conditional stimulus (CS) onset. Selecting this temporal region served two goals: (1) to exclude the initial nonstationary components of the brain response from the power spectrum; and (2) to emphasize ssVEP power in the second half of the CS presentation period, which has demonstrated greatest sensitivity to the conditioning procedure (Moratti and Keil, 2005; Moratti et al., 2006). The resulting posterior ssVEP amplitudes (in microvolts) were averaged across sensor 120 (just anterior to $\mathrm{Oz}$, in which ssVEP amplitude was maximal across participants) and its six immediate parieto-occipital neighbors (Electrical Geodesics sensors 111, 129, 128, 127, 119, and 110). A significance level of 0.05 (two-tailed) was used for all statistical analyses.

\section{Results}

To test the study hypotheses, we used a modified version of a conditional discrimination experiment, abbreviated as $\mathrm{AX}+1$ BX-(Jovanovic et al., 2005; Myers et al., 2009). To ensure that participants processed the cues as distinct elements and to check that participants showed evidence of fear discrimination, every trial within the conditioning phase was followed by an online rating of the likelihood ("not likely" to "likely") that each compound element would be followed by the UCS. Ratings collected during habituation served as the preconditioning baseline.

\section{Online CS ratings}

To validate our conditional discrimination design, we first examined participant CS ratings.

\section{Habituation phase}

Before the start of conditioning, there was no difference $(p>$ 0.28 ) in UCS expectancy after presentation of the A versus the B cue. Moreover, there were no differences between the A and X $(p>0.92)$ or B and $\mathrm{X}(p>0.80)$ elements.

\section{Conditioning phase}

Ratings for each of the respective cues in the AX+ and BX - pairs were binned (average of two trials per bin) and entered into two separate cue $(\mathrm{A} / \mathrm{X}$ and $\mathrm{B} / \mathrm{X}) \times$ bin (eight) repeated-measures ANOVAs. For the AX + trials, the analyses revealed an effect of cue $\left(F_{(1,20)}=38.41, p<0.001, \eta_{\mathrm{p}} 2=0.66\right)$ that was subsumed under a cue $\times$ bin interaction $\left(F_{(7,14)}=3.02, p=0.037, \eta_{\mathrm{p}} 2=\right.$ 0.60 ). As illustrated in Figure $2 A$, follow-up paired-sample $t$ tests for each bin indicated an increased expectancy of UCS presentation in response to the $\mathrm{A}+$ versus $\mathrm{X}$ cue for bins 2 through $8(p$ values $<0.02)$. These findings provide confirmatory evidence that participants were able to successfully discriminate the threatrelated cue when it was presented in compound with a neutral background (X) element.

In contrast, there were no main or interaction effects involving the $\mathrm{BX}-$ trials ( $p$ values $>0.11$ ), which were rated approximately equally unlikely to be followed by the UCS (Fig. $2 B$ ). As can be observed by comparing insets $A$ and $B$ in Figure 2, the $\mathrm{X}$ stimulus was rated as somewhat more likely to be followed by the UCS when paired with the threat $(\mathrm{A}+)$ cue than when paired with the safety $(\mathrm{B}-)$ cue. This visual impression was confirmed by a main effect $\left(F_{(1,20)}=13.36, p=0.002, \eta_{\mathrm{p}} 2=0.40\right)$ of cue $(\mathrm{X}$ from $\mathrm{A}+$ compound/X from $\mathrm{B}-$ compound), indicating that the $\mathrm{X}$ was rated differently depending on its association with either the threat or safety cue.

\section{ssVEP findings}

Figure 3 shows the Fourier frequency spectrum, collapsing across all participants, trial types, and experimental phases, indicating relative peaks at the two frequencies of steady-state stimulation (14 and $17.5 \mathrm{~Hz}$ ). Maximum strength of the ssVEP was concentrated over the posterior visual cortex as would be expected given the visual cortical sources giving rise to scalp recorded ssVEPs.

\section{Threat cue conditioning}

To assess the development of cortical perceptual biases during the excitatory component of fear conditioning, we conducted a cue $(\mathrm{A} / \mathrm{X}) \times$ learning phase (habituation, conditioning) $\times$ hemifield (left/right) repeated-measures ANOVA. This analysis revealed a significant cue $\times$ learning phase interaction $\left(F_{(1,19)}=6.48, p=\right.$ $\left.0.02, \eta_{\mathrm{p}} 2=0.25\right)$. Subsequent follow-ups, conducted separately 
A

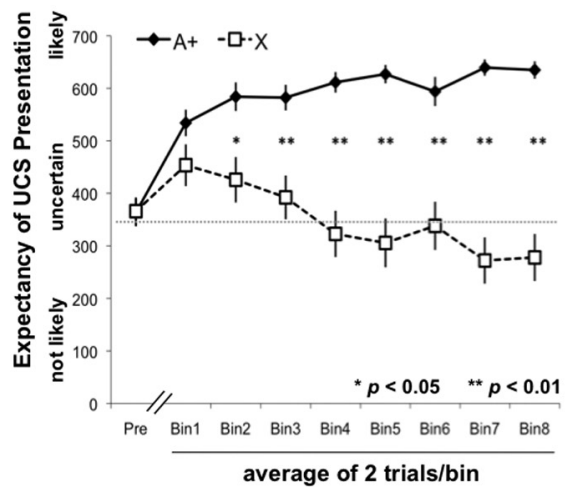

B

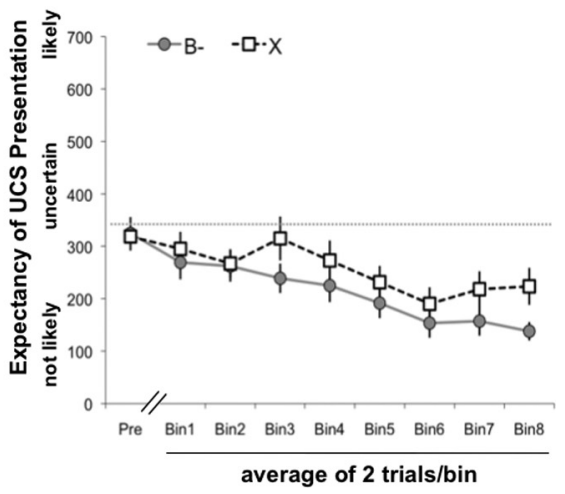

Figure 2. Online trial-by-trial ratings of $A X+(A)$ and $B X-(B)$ conditioned cues, before CS-UCS pairing (Pre) and during eight successive bins collected during the conditioning phase (each bin represents an average of 2 trials). The $y$-axis scale represents the horizontal dimension of the display monitor. The dashed gray line represents participants' uncertainty, with higher values indicating greater UCS expectancy and lower values indicating lower expectancy. Error bars depict SEM.

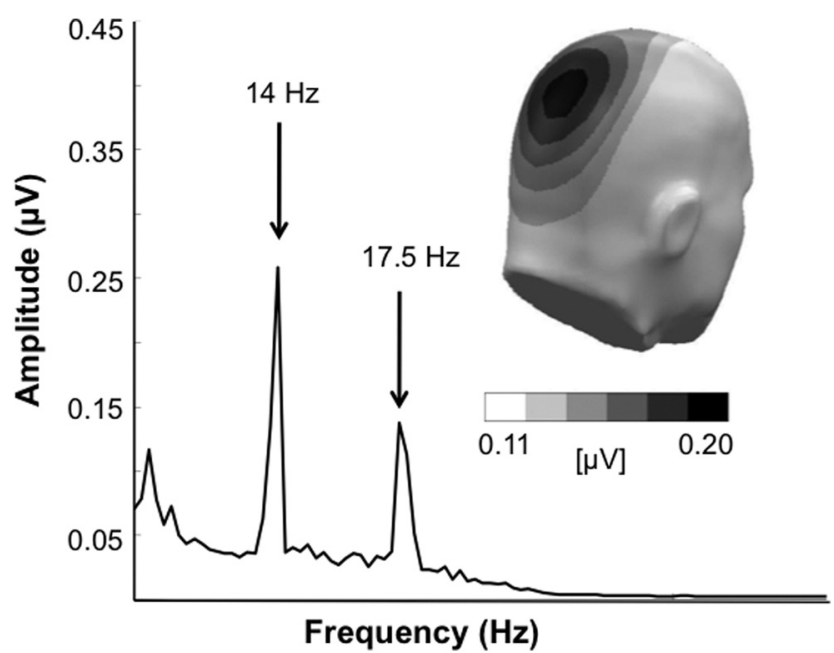

Figure 3. The Fourier frequency spectrum for Electrical Geodesics sensor 120, averaging across all subjects $(n=21)$ and experimental blocks. The grand average scalp topography of ssVEP amplitude to the conditional visual stimuli, collapsed across tagging frequencies.

for each learning phase, revealed no main effect of cue during the initial habituation phase $(F<1, p>0.36)$. However, during conditioning, the cue effect reached significance $\left(F_{(1,20)}=7.76\right.$, $\left.p=0.01, \eta_{\mathrm{p}} 2=0.28\right)$, reflecting increased ssVEP amplitude for the threat cue compared with the X (Fig. 4). Moreover, there was a significant increase in ssVEP amplitude from habituation to acquisition for the $\mathrm{A}(p=0.003)$ but not for the $\mathrm{X}(p>0.61)$ stimulus.

Because, as noted previously, the $\mathrm{X}$ grating was rated as having a different likelihood of being paired with the UCS depending on whether it was presented in compound with the threat or safety cue, we conducted a planned contrast examining ssVEP amplitude elicited by the background grating during the reinforced $\mathrm{AX}+$ and the unreinforced $\mathrm{BX}-$ compound trials. The analysis revealed no difference in ssVEP power evoked by the $\mathrm{X}$ when it was presented alongside the threat versus safety cue $(p>0.95)$.

\section{Safety cue conditioning}

A cue $(B / X) \times$ learning phase $\times$ hemifield ANOVA revealed no significant main or interaction effects $(p$ values $>0.14)$ for the safety conditioning component of the experiment. The cue $\times$ learning phase effect size $\left(\eta_{\mathrm{p}} 2=0.014\right)$ suggests that the null findings are most likely not attributable to low statistical power.

\section{Carrier frequency}

To ensure that the frequency tag assigned to each hemifield did not influence the results, flicker group (14 vs $17.5 \mathrm{~Hz}$ stimulation of left hemifield) was added as a between-subjects factor for the threat and safety conditioning analyses. Critically, there was no flicker group $\times$ cue $\times$ learning phase interaction ( $F$ values $<1$, $p$ values $>0.64$ ).

\section{Transfer tests}

The AB transfer test was conducted to provide an assessment of neural competition between learned threat and safety cues. This analysis revealed only a trend-level $\left(F_{(1,19)}=3.93, p=\right.$ $\left.0.06, \eta_{\mathrm{p}} 2=0.17\right)$ cue $(\mathrm{A} / \mathrm{B}) \times$ hemifield interaction, reflecting a marginally $(p=0.067)$ stronger ssVEP response to the threatrelated (mean \pm SEM, $0.28 \pm 0.05$ ) compared with the safety (mean \pm SEM, $0.22 \pm 0.03$ ) cue in the right hemifield. Critically, there was no significant $\left(p>0.48, \eta_{\mathrm{p}} 2=0.03\right.$ ) decrement in responding to the threat cue when it was presented in compound with a conditioned safety signal compared with the ssVEP strength achieved during the threat conditioning phase of the experiment (Fig. 5).

Analyses involving the AC transfer test, which examined competition between the threat cue and an associatively novel stimulus element, revealed no significant effects ( $p$ values $>0.084$ ). Importantly, there was no decrement in ssVEP amplitude for the $\mathrm{A}+$ element when it was paired with the novel $\mathrm{C}$ cue compared with the ssVEP amplitude attained during the threat conditioning $(p>0.52)$.

\section{Discussion}

The present study sought to answer two related questions: (1) what are the experience-dependent activity changes in human visual cortex during independent threat and safety signal conditioning? and (2) what are the sensory brain dynamics associated with responding to learned threat cues when they are viewed in the presence of co-occurring safety signals? To this end, we tracked cortical ssVEPs as participants performed a conditional discrimination task designed to isolate threat and safety components of aversive learning (Myers et al., 2009).

Online expectancy ratings indicated that participants were able to successfully discriminate discrete compound elements in terms of their association with an aversive outcome. In particular, the behavioral results provide evidence that the A+ grating acquired selective status as a threat cue and that the B cue functioned as a conditioned safety signal that predicted the absence of an aversive event. Our ssVEP findings support the hypothesis that the visual cortex is increasingly biased toward a simple low-level cue with an acquired threat value. The electrocortical results allow additional inferences regarding the specificity of conditioning effects, insofar as the increase in ssVEP amplitude was observed to be selective for the threat cue $(\mathrm{A}+)$ that was reinforced by the loud sound $100 \%$ of the time, without generalizing to the uninformative background stimulus (X). This observation is consistent with a previous MEG study using conditioned com- 
pound auditory stimuli, in which there was a relatively rapid (developing within five trials) discrimination between elements of the compound that were either informative or uninformative with respect to motivational reinforcement (Weisz et al., 2007). Because specificity is considered a criterion of associative learning (Weinberger, 2007), the pattern of our findings in which cortical vigilance was observed selectively for the threat cue argues against neuronal responses driven by general arousal that would have produced nonspecific vigilance. Although not a specific focus of the present paper, an inspection of the ssVEP data from the extinction phase of the experiment revealed that the ssVEP enhancement for the threat-related grating did not persist when participants were explicitly informed that loud noises would no longer be paired with the visual stimuli.

Although the background (X) grating was subjectively rated as more likely to be paired with the aversive sound when presented in combination with the reinforced threat signal compared with the trials in which it accompanied the safety cue, this was not reflected in a reliable difference in evoked oscillatory brain responses. The lack of ssVEP differentiation between the $\mathrm{X}$ grating on reinforced versus nonreinforced trials, despite a difference in subjective estimation, may pose a challenge to a straightforward interpretation of ssVEPs as tracking the development of a conditioned visuocortical bias. However, a closer examination of the ratings data revealed that the $\mathrm{X}$ grating was, on average, evaluated as having only an "uncertain" degree of association with the aversive noise, on the reinforced $\mathrm{AX}+$ compound trials, and that the X cue came to be evaluated as increasingly less likely to be the critical UCS predictor as training progressed.

Compared with the effects of threat conditioning, safety conditioning was not associated with increased ssVEP amplitudes in this experiment. We had expected the safety signal to become a salient perceptual feature and to elicit a conditioning-related ssVEP increase of greater amplitude compared with the neutral $(\mathrm{X})$ cues, because the safety cue is an indicator of "relief" from an aversive outcome (Lohr et al., 2007). Considerable evidence collected in human neuroscience and psychophysiological studies has demonstrated that environmental stimuli possessing intrinsic unpleasant and pleasant emotional qualities (e.g., images of injury or death or those portraying attractive nudes) lead to enhanced sensory processing when compared with the perception of neutral stimuli (Lang and Bradley, 2010). The

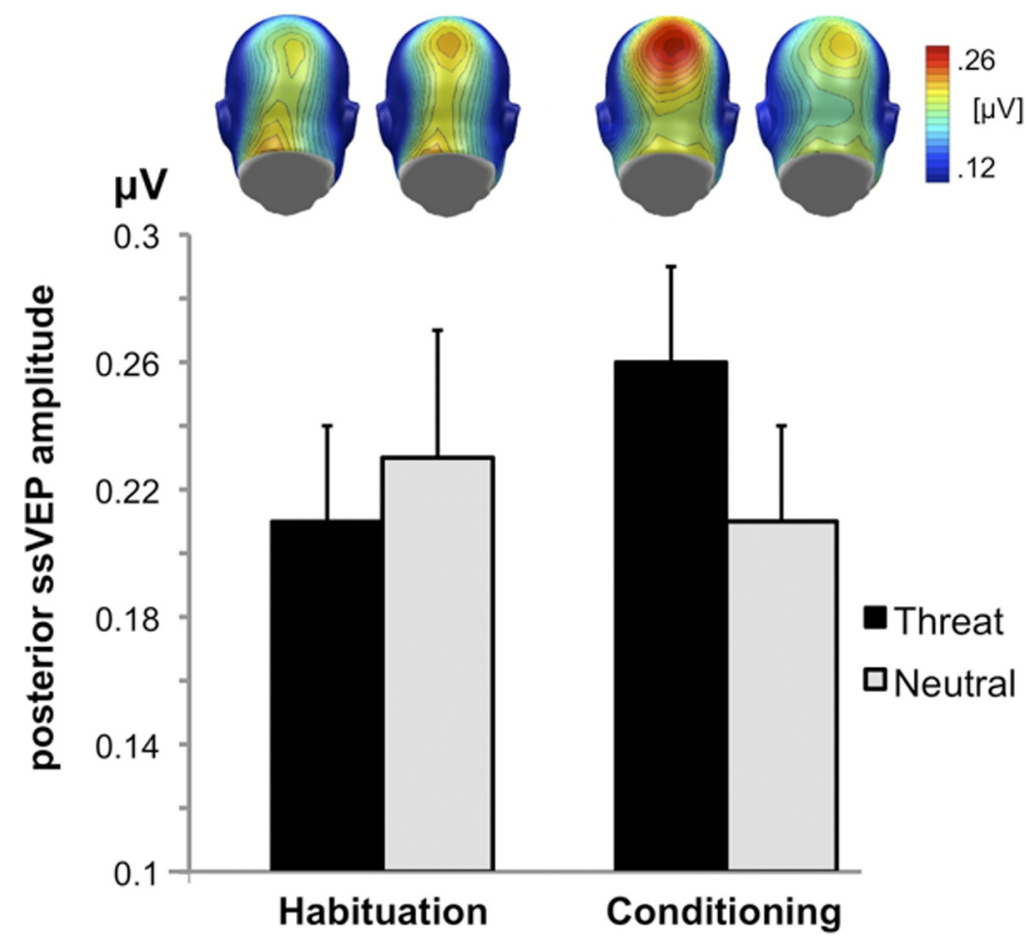

Figure 4. Means and scalp topographies (mapped with spherical spline interpolation) of ssVEP amplitude during threat cue conditioning, shown separately for the threat cue $(A+)$ paired with the UCS on $100 \%$ of the conditioning trials and the background stimulus (X) paired with the UCS on 50\% of the conditioning trials. Error bars depict SEM.

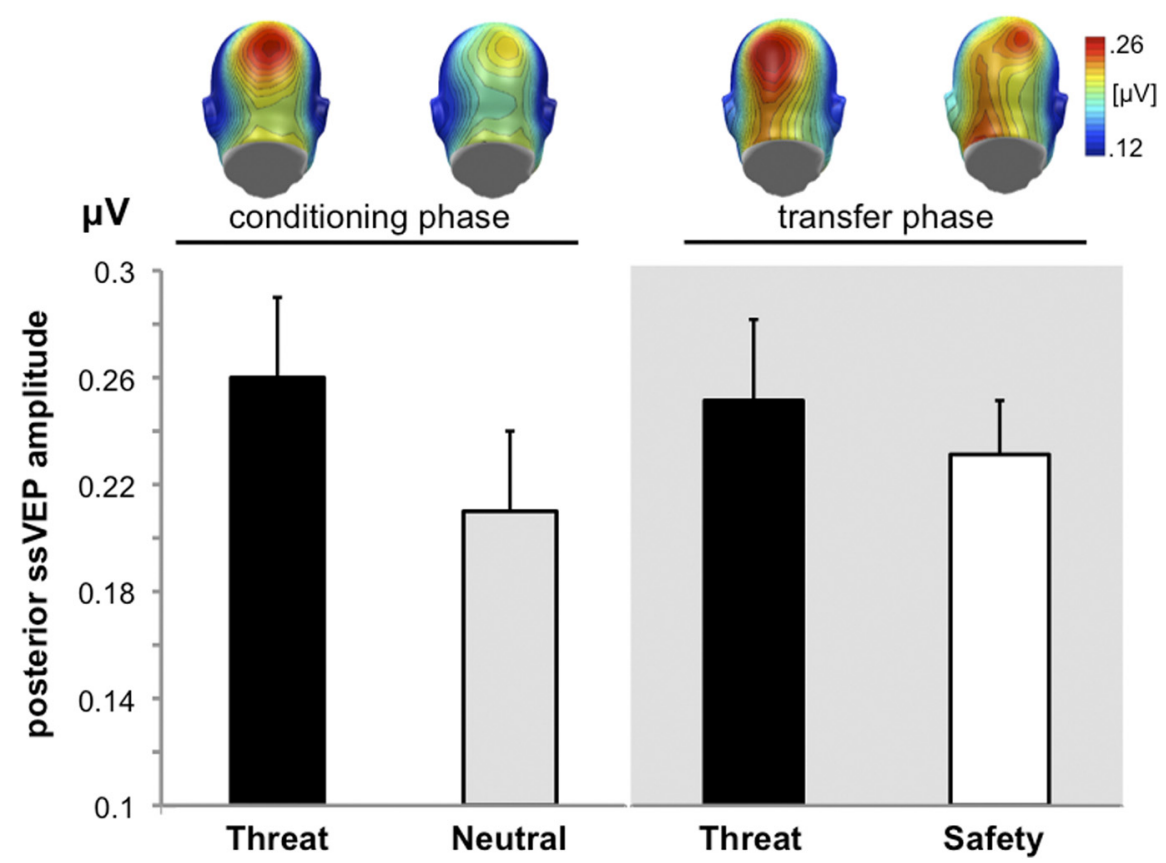

Figure 5. Means and scalp topographies (mapped with spherical spline interpolation) of sSVEP amplitudes evoked by the conditioned threat stimulus and a neutral background cue (conditioning phase, left) and that evoked by the threat and safety cues (transfer phase, right). Error bars depict SEM.

lack of safety signal ssVEP enhancement observed here suggests that a learned cue that reliably signals the omission of an aversive event is not necessarily salient enough to produce a selective increase in the engagement of cortical sensory networks. In this regard, our finding may be comparable with previous evidence from neuromagnetic responses to non-reinforced CS- cues in 
differential conditioning paradigms (Moratti and Keil, 2005; Moratti et al., 2006), although those studies were not explicitly designed to isolate threat and safety learning and did not include non-predictive cues as components of a compound. Some have suggested that the time course of inhibitory learning and its expression may be slower compared with excitatory learning, because the former may consume more processing resources and require reentry from more extensive brain structures (Weike et al., 2008). However, we consider this to be an unlikely explanation for our findings because the present experimental design involved considerably more safety training trials than were used in previous research (Jovanovic et al., 2005). Moreover, the online ratings indicate that participants already learned the negative relationship between the presence of the safety cue and aversive outcomes within the first two learning trials, that is, they were cognitively aware that the safety cue consistently signaled absence of an aversive outcome.

Simultaneous presentation of learned threat and safety signals (in the absence of reinforcement) did not diminish visuocortical responding to the threat cue, as measured by ssVEPs. These findings complement previous work in humans (Jovanovic et al., 2005) and other animals (Myers and Davis, 2004; Winslow et al., 2008), demonstrating decreased fear-potentiated startle reflex magnitudes when conditioned threat and safety cues were presented in compound. The intact visuocortical response bias during the transfer test stage suggests that it may be expedient to continue allocating perceptual and attentional resources to cues associated with threat in previous encounters, even when competing information about safety is available and when aversive reinforcement is discontinued. Considered from a functional perspective, remaining perceptually vigilant to potential threat in the environment seems adaptive, despite the simultaneous presence of conflicting information (i.e., one errs on the side of caution).

The present results highlight the differences between the sensory aspects of threat processing in the brain compared with the preparation and execution of action at the peripheral level (Lang et al., 1997): somatic activation of defensive reflexes may be relaxed when conflicting threat-safety cues are simultaneously present (Jovanovic et al., 2005), indicating a strategic difference between cortical sensory and peripheral/behavioral responding in situations of uncertain threat. To return to a previous example, cortical sensory systems appear to remain alert to features of the environment that have predicted potentially harmful outcomes in one's previous experience (e.g., growling dogs), even if overt mobilization of defensive responses is inhibited by information indicating safety (e.g., the presence of a leash). This hypothesis can be explicitly tested in the future by experiments that incorporate measures of defensive somatic reflexes and cortical perceptual processing in a single study design. Another difference worth noting is that the fear-potentiated startle represents a global response to threat-safety compound presentations, whereas the frequency-tagged EEG responses measured in this study allowed us to investigate separate responses to each individual cue. The capacity to isolate specific responses to discrete cues presented in compound is a unique property of entrained brain oscillatory measures.

Our finding of a threat-related cortical response bias, unperturbed by the presence of stimuli indicating safety or associative novelty, appears approximately analogous to previous evidence from our laboratory that socially anxious individuals exhibit enhanced electrocortical responding to threatening faces without competition from simultaneously presented friendly or neutral expressions (Wieser et al., 2011). It is possible that the spatial arrangement of stimuli in both of these studies accounts for the lack of competition, because previous ssVEP research has suggested that processing resources can be allocated across noncontiguous regions of the visual field (Müller et al., 2003), i.e., those separated by more than $\sim 4.5^{\circ}$ of visual angle. In the future, ssVEP designs that involve a full spatial overlap of conditioned threat and safety cues may prove to have increased sensitivity for revealing competitive excitatory-inhibitory interactions.

It should be mentioned that the current study was specifically designed to examine biases in visuocortical processing of conditioned stimuli, by exploiting the unique properties of frequencytagged ssVEP responses. It remains plausible that neural activity and/or functional connectivity in extended defensive neuronal networks (e.g., amygdala, insula) undergoes modification when responding to learned threat signals in the presence of safety information or when conditioned threat cues become predictive of safety during subsequent learning stages (Schiller et al., 2008). Answers to these questions may carry considerable implications for our future understanding of both normative fear suppression mechanisms and their dysfunction in clinical fear disorders (Mineka and Oehlberg, 2008). To this end, an extension of the present paradigm to other neuroimaging modalities and with larger sample sizes would contribute to a better understanding of the neurophysiological changes accompanying threat and safety learning.

\section{References}

Andersen SK, Müller MM (2010) Behavioral performance follows the time course of neural facilitation and suppression during cued shifts of featureselective attention. Proc Natl Acad Sci U S A 107:13878-13882. CrossRef Medline

Armony JL, Dolan RJ (2002) Modulation of spatial attention by fear conditioned stimuli: an event-related fMRI study. Neuropsychologia 40:817826. CrossRef Medline

Brainard DH (1997) The psychophysics toolbox. Spat Vis 10:433-436. CrossRef Medline

Damaraju E, Huang YM, Barret LF, Pessoa L (2009) Affective learning enhances activity and functional connectivity in early visual cortex. Neuropsychologia 47:2480-2487. CrossRef Medline

Jovanovic T, Keyes M, Fiallos A, Myers KM, Davis M, Duncan EJ (2005) Fear potentiation and fear inhibition in a human fear-potentiated startle paradigm. Biol Psychiatry 57:1559-1564. CrossRef Medline

Junghöfer M, Elbert T, Tucker DM, Rockstroh B (2000) Statistical control of artifacts in dense array EEG/MEG studies. Psychophysiology 37:523532. CrossRef Medline

Keil A, Gruber T, Müller MM, Moratti S, Stolarova M, Bradley MM, Lang PJ (2003) Early modulation of visual perception by emotional arousal: Evidence from steady-state visual evoked brain potentials. Cogn Affect Behav Neurosci 3:195-206. CrossRef Medline

Keil A, Stolarova M, Moratti S, Ray WJ (2007) Adaptation in human visual cortex as a mechanism for rapid discrimination of aversive stimuli. Neuroimage 36:472-479. CrossRef Medline

Kluge C, Bauer M, Leff AP, Heinze HJ, Dolan RJ, Driver J (2011) Plasticity of human auditory-evoked fields induced by shock conditioning and contingency reversal. Proc Natl Acad Sci U S A 108:12545-12550. CrossRef Medline

Lang PJ, Bradley MM (2010) Emotion and the motivational brain. Biol Psychol 84:437-450. CrossRef Medline

Lang PJ, Bradley MM, Cuthbert BN (1997) Motivated attention: affect, activation, and action. In: Attention and orienting: sensory and motivational processes (Lang PJ, Simons RF, Balaban MT, eds), pp 97-135. Hillsdale, NJ: Erlbaum.

Lohr JM, Olatunji BO, Sawchuk CN (2007) A functional analysis of danger and safety signals in anxiety disorders. Clin Psychol Rev 27:114-126. CrossRef Medline

Mineka S, Oehlberg K (2008) The relevance of recent developments in classical conditioning to understanding the etiology and maintenance of anxiety disorders. Acta Psychol (Amst) 127:567-580. CrossRef Medline 
Moratti S, Keil A (2005) Cortical activation during Pavlovian fear conditioning depends on heart rate response patterns: an MEG study. Brain Res Cogn Brain Res 25:459-471. CrossRef Medline

Moratti S, Keil A (2009) Not what you expect: experience but not expectancy predicts conditioned responses in human visual and supplementary cortex. Cereb Cortex 19:2803-2809. CrossRef Medline

Moratti S, Keil A, Miller GA (2006) Fear but not awareness predicts enhanced sensory processing in fear conditioning. Psychophysiology 43: 216-226. CrossRef Medline

Morris JS, Friston KJ, Dolan RJ (1998) Experience-dependent modulation of tonotopic neural responses in human auditory cortex. Proc Biol Sci 265:649-657. CrossRef Medline

Müller MM, Teder-Sälejärvi W, Hillyard SA (1998) The time course of cortical facilitation during cued shifts of spatial attention. Nat Neurosci 1:631-634. CrossRef Medline

Müller MM, Malinowski P, Gruber T, Hillyard SA (2003) Sustained division of the attentional spotlight. Nature 424:309-312. CrossRef Medline

Myers KM, Davis M (2004) AX+, BX-discrimination learning in the fearpotentiated startle paradigm: possible relevance to inhibitory fear learning in extinction. Learn Mem 11:464-475. CrossRef Medline

Myers KM, Toufexis DJ, Winslow JT, Jovanovic T, Norrholm SD, Duncan E, Davis M (2009) Measurement of fear inhibition in rats, monkeys, and humans with and without posttraumatic stress disorder, using the AX+, BX - paradigm. In: The human amygdala (Whalen PJ, Phelps EA, eds), pp 61-81. New York: Guildford.

Padmala S, Pessoa L (2008) Affective learning enhances visual detection and responses in primary visual cortex. J Neurosci 28:6202-6210. CrossRef Medline

Pelli DG (1997) The VideoToolbox software for visual psychophysics: transforming numbers into movies. Spat Vis 10:437-442. CrossRef Medline

Peyk P, De Cesarei A, Junghöfer M (2011) ElectroMagnetoEncephalogra- phy software: overview and integration with other EEG/MEG toolboxes. Comput Intell Neurosci 2011:861705. CrossRef Medline

Schiller D, Levy I, Niv Y, LeDoux JE, Phelps EA (2008) From fear to safety and back: reversal of fear in the human brain. J Neurosci 28:11517-11525. CrossRef Medline

Stolarova M, Keil A, Moratti S (2006) Modulation of the C1 visual eventrelated component by conditioned stimuli: evidence for sensory plasticity in early affective perception. Cereb Cortex 16:876-887. CrossRef Medline

Vialatte FB, Maurice M, Dauwels J, Cichocki A (2010) Steady-state visually evoked potentials: focus on essential paradigms and future perspectives. Prog Neurobiol 90:418-438. CrossRef Medline

Wang J, Clementz BA, Keil A (2007) The neural correlates of feature-based selective attention when viewing spatially and temporally overlapping images. Neuropsychologia 45:1393-1399. CrossRef Medline

Weike AI, Schupp HT, Hamm AO (2008) In dubio pro defensio: initial activation of conditioned fear is not cue specific. Behav Neurosci 122: 685-696. CrossRef Medline

Weinberger NM (2007) Associative representational plasticity in the auditory cortex: a synthesis of two disciplines. Learn Mem 14:1-16. CrossRef Medline

Weisz N, Kostadinov B, Dohrmann K, Hartmann T, Schlee W (2007) Tracking short-term auditory cortical plasticity during classical conditioning using frequency-tagged stimuli. Cereb Cortex 17:1867-1876. CrossRef Medline

Wieser MJ, McTeague LM, Keil A (2011) Sustained preferential processing of social threat cues: bias without competition? J Cogn Neurosci 23:19731986. CrossRef Medline

Winslow JT, Noble PL, Davis M (2008) AX+/BX- discrimination learning in the fear-potentiated startle paradigm in monkeys. Learn Mem 15:63-66. CrossRef Medline 